\title{
Efficient Time-Domain Analysis of Highly-Dispersive Linear and Non-Linear Metamaterial Waveguide and Antenna Structures operated in the Impulse-Regime
}

\author{
J. S. Gómez-Díaz, S. Gupta, A. Alvarez-Melcon and C. Caloz
}

\begin{abstract}
A numerical method is proposed for the analysis of highly-dispersive linear and non-linear metamaterial structures. Firstly, pulse propagation along non-linear composite right/left-handed transmission line (CRLH TL) is studied. The dispersive CRLH line is loaded with hyper abrupt diodes to achieve non-linearity. The characteristic impedance and propagation constant of each unit cell of the CRLH TL is varied at each time-step, and the non-uniform line is then analyzed. A novel interpolation scheme, based on the smooth behaviour of the propagation constant with small variations of the shunt capacitor is proposed. Numerical results show that high accuracy and up to $80 \%$ reduction of the computational cost is achieved. A general example which combines dispersion and non-linearity is presented, validated and discussed. Secondly, the impulse-regime radiation of a leaky-wave antenna is analyzed and used to describe a real-time spectrogram analyzer (RTSA). A new calibration method for the system, which takes into account the directivity variation of the antenna with frequency, is proposed. This method provides an extremely fast and accurate tool to characterize the behaviour of leaky-wave based RTSA systems.
\end{abstract}

\section{Introduction}

The analysis of highly-dispersive structures, such as transmission lines, waveguides or antennas, has usually been performed in the harmonic regime. These structures have normally been employed in the non-dispersive frequency region, especially at the microwave domain. However, their inherent dispersive features may be exploited at the impulse-regime, in order to obtain novel and exotic applications in the area of Ultra-Wideband (UWB) communications [1], radars [2], Electromagnetic Interference and Compatibility (EMI/EMC), security or instrumentation. Specifically, the introduction of metamaterials $[3,4]$ in the last decade has provided novel broadband dispersive effects at the microwave domain, which may contribute to the development of novel applications, such as [5] or [6]. Furthermore, metamaterial lines can easily be loaded with non-linear elements, leading to harmonic-generation in the impulse regime. The combination of both effects, dispersion and non-linearity, provides a wide range of optics-inspired phenomena [7] at microwaves, such as the soliton [8,9] (a wave able to propagate without any distortion along dispersive non-linear structures).

The temporal analysis of highly-dispersive linear metamaterial structures can be performed with time-domain techniques, such as FDTD [10] or TLM [11]. However, these accurate methods require a high computational cost, due to the meshing of the whole geometry under study. In addition, when the dispersive structures also include non-linear elements (such as varactors) this analysis is much more complicated. An interesting alternative is the transmission line approach based on time-domain Green's functions. This technique was recently applied to the temporal analysis of dispersive metamaterials structures [12]. With this equivalent transmission line simplification of the geometry, the Green's functions are available in closed-form, and directly correspond to the voltages and currents along the transmission line. The main advantages of this approach are the unconditional stability and fast computation, due to 
the continuous treatment of time, and the insight into the physical phenomena provided by the Green's functions.

In this paper, we propose a numerical method [13] to study: (a) impulse-regime phenomenology of non-linear metamaterial composite right/left-handed (CRLH) transmission lines (TL) [14], and (b) radiative impulse-regime properties of CRLH leaky-wave antennas (LWAs) [3] and its application to the characterization of a realtime spectrogram analyzer (RTSA) $[15,16]$.

In the case (a), non-linearity is achieved by replacing the shunt capacitor of the CRLH TL unit cell by a varactor [13]. For the proposed non-linear analysis, time discretization is required. Specifically, the characteristic impedance and propagation constant are re-evaluated at each time for each unit cell of the CRLH TL. As a difference of [13], the variation of the characteristic impedance of each unit cell is considered, introducing a temporal-dependent mismatch, correctly modelling the nonlinear propagation of the pulse. These impedance variations create small reflected waves between two consecutive unit cells, which were not present in the model implemented in [13]. A non-uniform transmission line is therefore obtained and solved at each particular instant. Then, a novel interpolation scheme has been developed to reduce the computational cost required for the technique. The idea is to interpolate the propagation constant value as a function of the variable shunt capacitor. Finally, a general example, where the dispersive effects are combined with the non-linear phenomena, is presented and discussed.

In the case (b), the radiation formulation presented in [13] has been employed to characterize a real-time spectrogram analyzer (RTSA) system $[15,16]$, based on CRLH leaky-wave antennas (LWAs). A novel power calibration method has been proposed to accurately characterize unknown signals, considering the directivity variation with frequency. This method provides an extremely fast tool to design a RTSA system and to determine the range of valid input signals that can be characterized. The computational cost is reduced to the orders of seconds, as compared with the hours required with the full-wave analysis employed in $[14,15]$.

\section{Time-Domain Transmission Line Response}

In this section, a time-domain Green's function approach is employed to determine the transmission line response in the impulse-regime. For this purpose, we initially consider the field radiated by a distributed source in an arbitrary (possible dispersive and anisotropic) homogeneous medium as a function of time [17], which can be computed by

$$
\vec{E}(\vec{r}, t)=\frac{-\mu_{0}}{2 \pi} \iiint \overline{\overline{\tilde{G}}}\left(\vec{r}, \vec{r}^{\prime} ; \omega\right) \cdot \vec{J}\left(\vec{r}^{\prime}, t^{\prime}\right) e^{j \omega\left(t-t^{\prime}\right)} \partial r^{\prime} \partial t^{\prime} \partial \omega,
$$

where $\vec{r}$ is an observation point in the medium, $\vec{r}^{\prime}$ is the position of the distributed source and $\vec{J}\left(\vec{r}^{\prime}, t^{\prime}\right)$ provides the temporal evolution of the input signal as a function of space.

In the case of a transmission line (1D), the source may be considered punctual and located at $\vec{r}_{g}$ [i.e. $J\left(\vec{r}^{\prime}, t^{\prime}\right)=\delta\left(r_{g}\right) I\left(t^{\prime}\right)$ ], the dyadic variables are automatically converted to scalar, and therefore Eq. (1) reduces to [12]

$$
X(\vec{r}, t)=\frac{-\mu_{0}}{2 \pi} \int \tilde{G}_{X}\left(\vec{r}, \vec{r}_{g} ; \omega\right) \tilde{I}(\omega) e^{j \omega t} \partial \omega
$$

where $X(\vec{r}, t)$ represents the voltage or current along the transmission line and $\tilde{I}(\omega)$ is the Fourier transform of the input waveform. 
Since the temporal evolution of the source is known, only the Green's function of the line needs to be calculated. Specifically, in this work artificial metamaterial composite right/left-handed (CRLH) transmission lines [3] are considered. These lines are broadband and dispersive in nature, and may host novel interesting phenomena and applications in the impulse-regime.

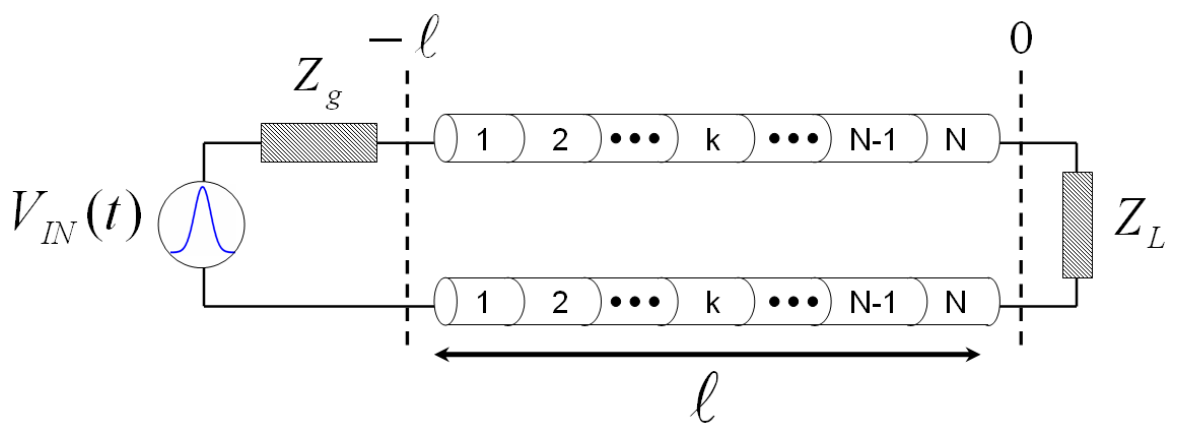

a)

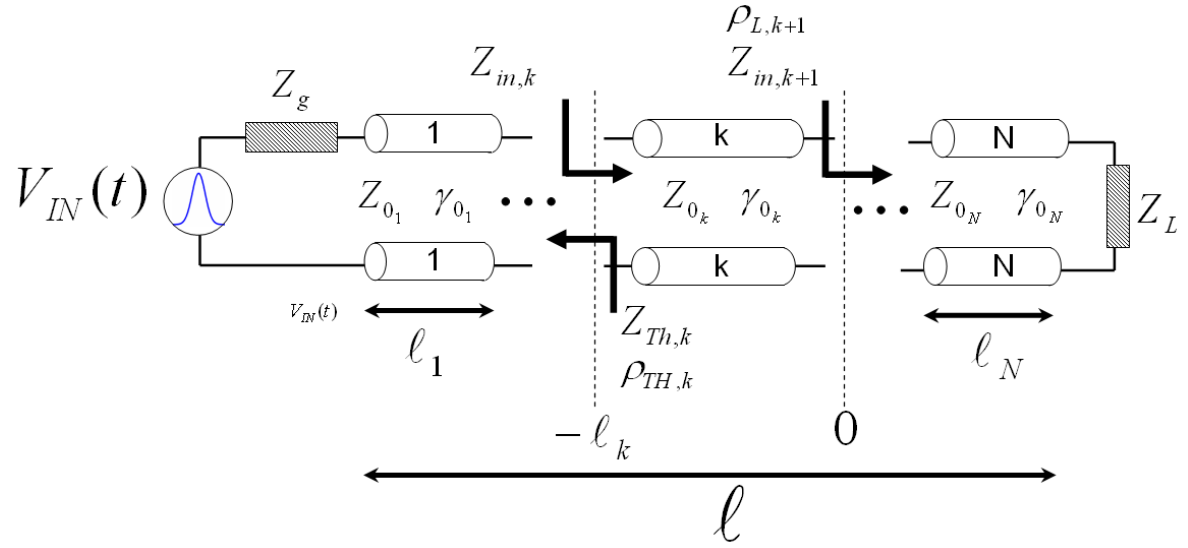

b)

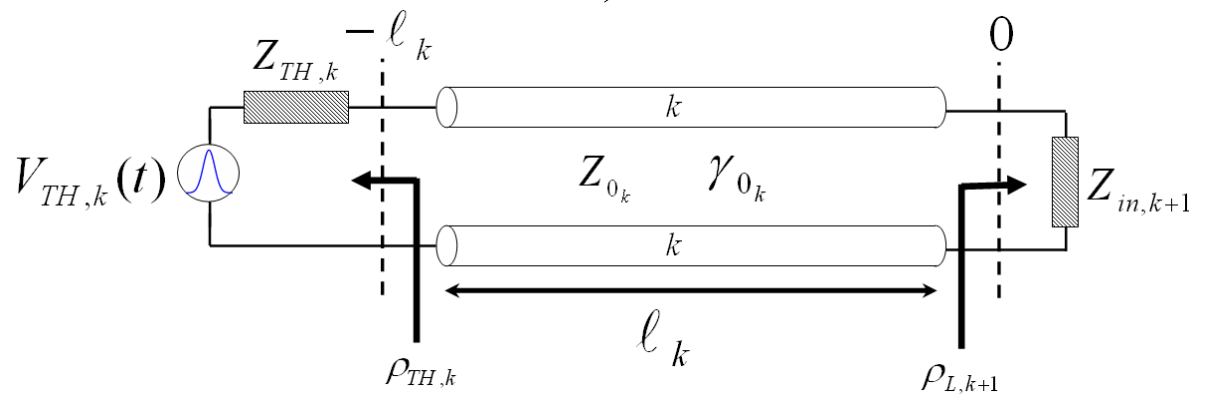

c)

Figure 1: Dispersive artificial transmission line excited by a point source generator. (a) Uniform case. The line, composed of $\boldsymbol{N}$ unit cells, is defined by its characteristic impedance $\left(Z_{0}\right)$, propagation constant $\left(\gamma_{C}\right)$ and length $(\ell)$. (b) Non-uniform case. The line is composed of $N$ uniform transmission line sections. Each $k^{\text {th }}$ section has its own length $\left(\ell_{k}\right)$, characteristic impedance ( $Z_{0_{K}}$ ) and propagation constant ( $\gamma_{C_{K}}$ ). (c) Thévenin equivalent circuit for the k-th uniform transmission line section.

In the simplest case of a uniform and matched line [see Fig. 1a)], which is composed of $N$ unit cells, the Green's function of the line reduces to [12] 


$$
\begin{aligned}
& \overline{\overline{\tilde{G}}}_{V}\left(\vec{r}, \vec{r}_{g} ; \omega\right)=e^{-\gamma(\omega) R_{\ell}} \\
& \overline{\overline{\tilde{G}}}_{I}\left(\vec{r}, \vec{r}_{,} ; \omega\right)=\frac{e^{-\gamma(\omega) R_{\ell}}}{Z_{0}(\omega)}
\end{aligned}
$$

where $R_{\ell}=\left|z-z_{g}\right|$ is the distance between the generator point source $\left(z_{g}\right)$ and the observer position on the line $(z), Z_{0}(\omega)$ is the characteristic impedance of the line [3] and $\gamma(\omega)$ is the propagation constant.

In a general non-uniform line, each unit cell of the line may have different parameters. In this case, reflections occur due to the transition between two consecutive cells and a different propagation conditions appear at each cell [see Fig.1 b)]. . The Green's function along the $k_{t h}$ uniform transmission line section $\left(z \in\left[-\ell_{k}, 0\right]\right)$, including generator and load mismatches reads $[18,19]$

where

$$
G_{V}\left(z, z^{\prime}=-\ell ; \omega\right)=A(\omega)\left[e^{-\gamma_{k}(\omega) z}+\rho_{L, k+1}(\omega) e^{\gamma_{k}(\omega) z}\right]
$$

$$
A(\omega)=\frac{V_{T H, k}(\omega) Z_{i n, k}}{Z_{i n, k}(\omega)+Z_{T H, k}(\omega)} \frac{e^{-\gamma_{k}(\omega) \ell_{k}}}{1-\rho_{L, k+1}(\omega) \rho_{T H, k}(\omega) e^{-2 \gamma_{k}(\omega) \ell_{k}}},
$$

with

$$
\rho_{T H, k}(\omega)=\frac{Z_{T H, k}(\omega)-Z_{0_{k}}(\omega)}{Z_{T H, k}(\omega)+Z_{0_{k}}(\omega)}
$$

and

$$
\rho_{L, k}(\omega)=\frac{Z_{i n, k+1}(\omega)-Z_{0_{k}}(\omega)}{Z_{i n, k+1}(\omega)+Z_{0_{k}}(\omega)} .
$$

which was obtained by equating the Green's function evaluated at the input of the $k_{\text {th }}$ section, to the Thevenin's voltage evaluated at the same section $V_{T H, k}$ [see Fig.1 c) ]. By recurrently applying Eq. (5), the voltage as a function of time may be computed at any point along the nonuniform transmission line.

\section{Linear and Non-Linear Metamaterial Guiding Structures}

Let us discuss pulse propagation along a CRLH TL considering two different unit-cell configurations, linear and non-linear.

In the linear case, the parameters of the unit cell are constant with time, as can be observed in Fig. 2a). Therefore, all unit cells behave as a single medium, without any reflection among the cells. For pulse propagation, it is particularly interesting to design the line in a balanced condition, i.e. mutual cancellation between series and shunt resonances, leading to a broadband operation. In this case, the computation of the complex propagation constant is simplified to [3]:

$$
\gamma(\omega)=\frac{1}{p} \cosh ^{-1}\left(1-\frac{\chi(\omega)}{2}\right),
$$

with

$$
\chi(\omega)=\left(\frac{\omega}{\omega_{R}}\right)^{2}+\left(\frac{\omega_{L}}{\omega}\right)^{2}-\kappa \omega_{L}^{2}
$$


and

$$
\kappa=L_{R} C_{L}+L_{L} C_{R},
$$

where $\omega_{R}=1 / \sqrt{L_{R} C_{R}}, \omega_{L}=1 / \sqrt{L_{L} C_{L}}$ and $\mathrm{p}$ is the unit cell's length.

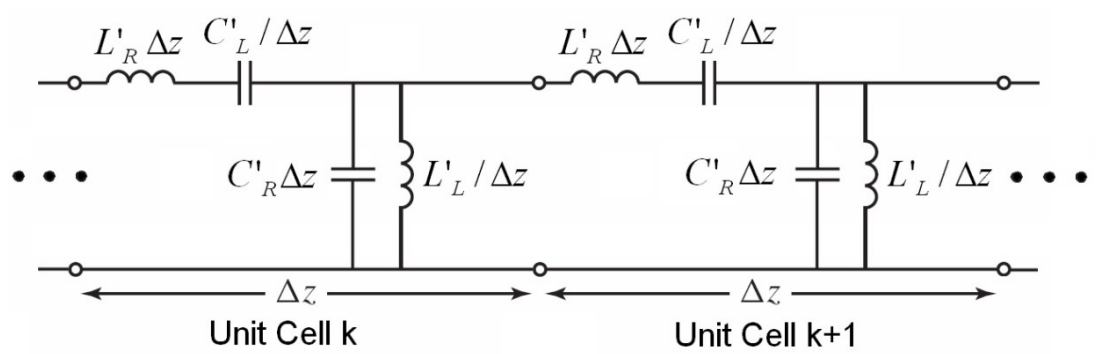

a)

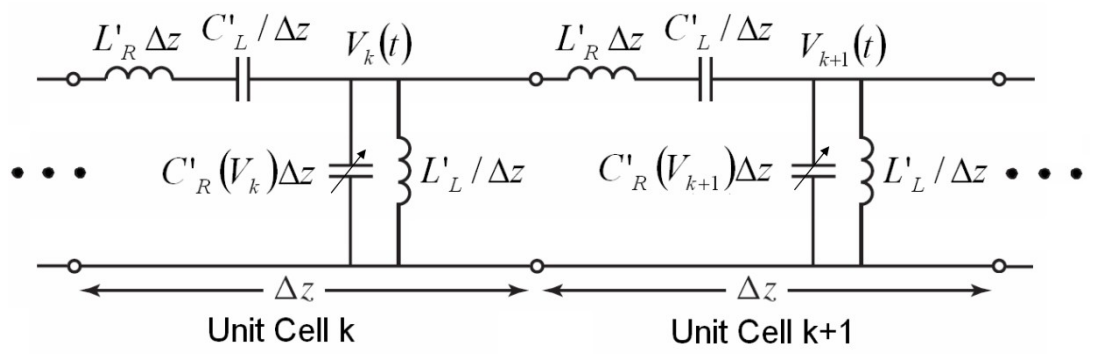

b)

Figure 2: Equivalent circuit model for the CRLH unit cells $k^{\text {th }}$ and $(k+1)^{t h}$. (a) Linear case. (b) Non-linear case, where the capacitor $C^{\prime}{ }_{R}$ has been replaced by a hyper abrupt diode.

In the non-linear case, the parameters of the unit cell vary with time. This is modelled by replacing the shunt capacitors $\left(C_{R}\right)$ by hyper-abrupt junction diodes, which provides a capacitor value function of the voltage applied at their terminals [see Fig. 2b)]. Specifically, this type of varactors exhibit the following C-V law

$$
C_{R}(V)=\frac{C}{\left(1+V / V_{0}\right)} \approx C_{0}+\eta V+\alpha V^{2}
$$

where $V$ is the voltage at the varactor terminals, and $V_{0}$ is the applied bias voltage.

In order to analyze this non-linear line, a time-discretization is required. At the initial time $\left(t=t_{a}\right)$ a boundary condition of $0 \mathrm{~V}$ value along the non-linear line is imposed [see Fig. 3a)]. Then, at any other time $\left(t=t_{b}\right)$ the $C_{R}$ parameter of the $k_{t h}$ unit-cell varies as a function of the voltage present in the previous time at that node [ $V_{k}\left(t_{b-1}\right)$ ], which results into an effectively non-uniform medium [see Fig. 3b)]. This non-uniform medium can be efficiently analyzed with the method presented in the previous section. 


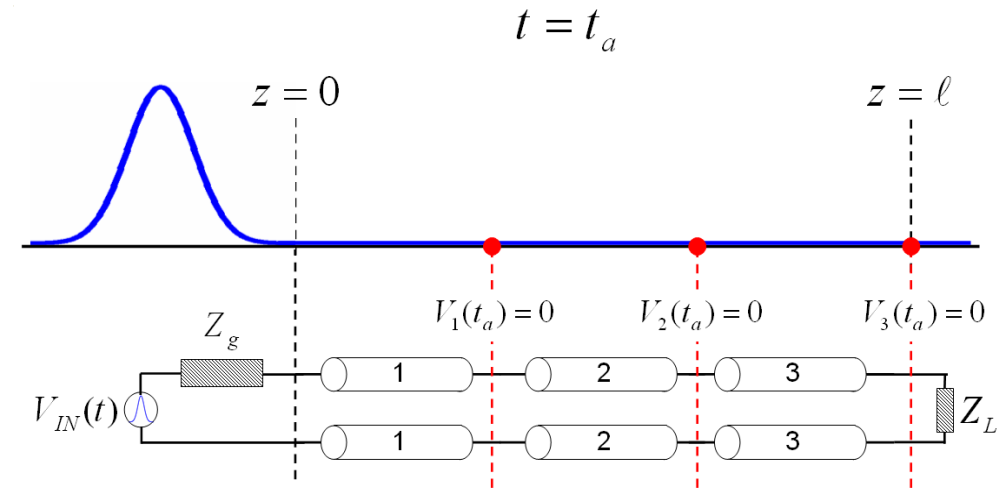

a)

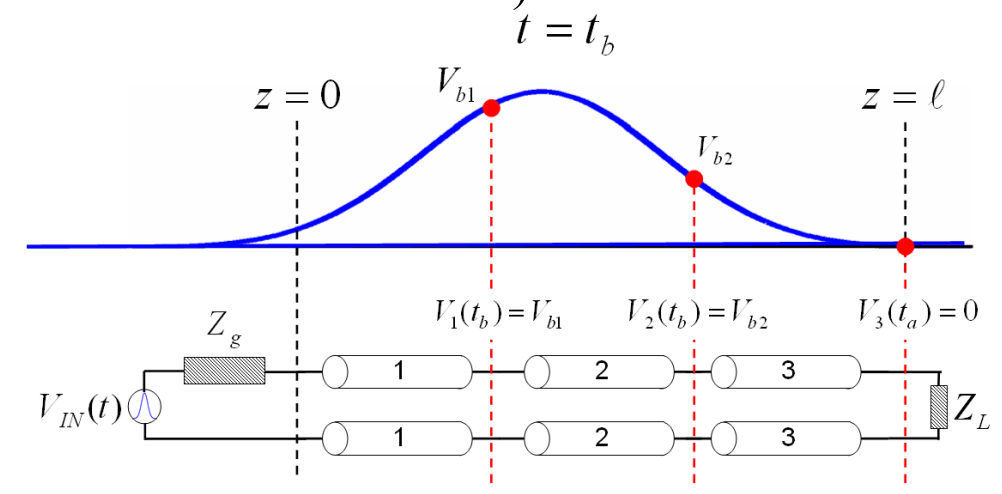

b)

Figure 3: Pulse propagation along a non-linear CRLH transmission line composed of 3 unit cells. The voltage at each unit cell node controls the non-linear behaviour of the line. (a) Initial situation, where the time boundary condition imposes a 0 voltage at all unit cell nodes. (b) General situation, where a different voltage is applied to each unit cell node.

Thereby, a new propagation constant and a new characteristic impedance must now be computed for each unit cell at any discrete instant of time. The variation of the characteristic impedance creates reflected waves among the unit cells. These small reflected waves can be observed in the results that will be presented later in this section, and they were not present with the simpler model used in [13]. Moreover, note that the balanced assumption made for the linear case does not hold any more at all times, due to the time-variation of the shunt capacitor. Therefore, the complex propagation constant must be computed using the scattering parameters of each unit cell [3], unwrapping the phase of the transmission parameter as follows

$$
\gamma(\omega)=\alpha(\omega)+j \beta(\omega)=-\frac{\ln \left|S_{21}\right|}{\ell}+j\left[2 \pi \mathrm{m}-\phi^{\text {unwrapped }}\left(S_{21}\right)\right],
$$

where $\mathrm{m}$ is an integer [3].

It is important to mention that the proposed approach is based on the weak-non linearity assumption [8]. As commented, the method assumes that the media is linear at each particular instant. In addition, it also assumes that the variation of the media features (i.e. the influence of the non-linearity) is weak. Therefore, a smooth variation of the unit-cells parameters is required. Then, the use of Eq.(2) recovers the temporal evolution of the pulse as it propagates along the line, including the weak non-linear effects. Note that this approach is not appropriate for modelling strong non-linear lines, because in that case the variation of the unit-cell features may be abrupt, and the assumption that the medium is linear at particular instant may not hold any more [8]. 
This iterative process produces a significant increase in the computational cost as compared to the linear case. In order to reduce it, an interpolation scheme for the computation of the propagation constant is proposed. It is also based on the weak nonlinearity assumption [8], which is commonly employed to model non-linear media. Since the input pulse amplitude is known a priori, the possible variations of the shunt capacitor $C_{R}$ occur in a well-defined range. Under these conditions, the propagation constant of a single unit cell presents a smooth behaviour as a function of $C_{R}$, as shown in Fig. 4, and can be efficiently interpolated for an arbitrary $C_{R}$ value. This simple method provides an important computational cost reduction while maintaining high accuracy.

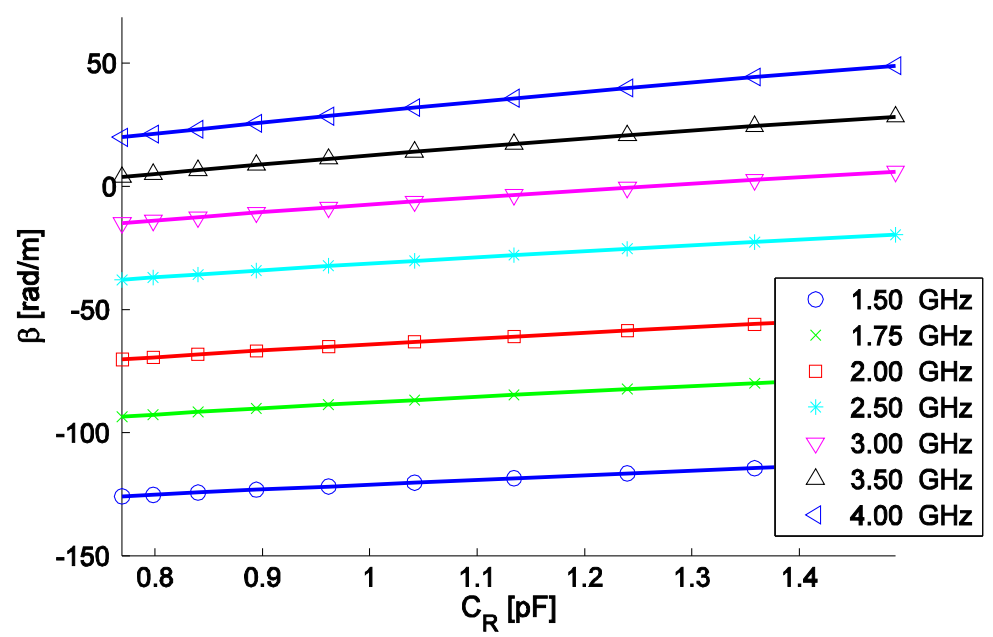

Figure 4: Propagation constant evolution versus the non-linear $C_{R}$ capacitor, plotted at different frequencies for a single CRLH unit cell. The cell parameters are $C_{0}=C_{L}=1.0 \mathrm{pF}$ and $L_{R}=L_{L}=2.5 \mathrm{nH}$.

The complete iterative process proposed to model pulse propagation along a weak non-linear CRLH transmission line is presented in Fig. 5. As can be observed in the flowchart, an analysis of the non-uniform line is performed at each time step. The maximum duration of this step is related to the input signal as

$$
\Delta t=\frac{1}{2 \mathrm{~N}\left(f_{0}+{ }^{B W} / 2\right)},
$$

where $f_{0}$ and $B W$ are the modulation frequency and the bandwidth of the input pulse, respectively. The $N$ parameter (with $N>=1$ ) controls the accuracy of the results, and the number of harmonics which can be recovered using this technique. 


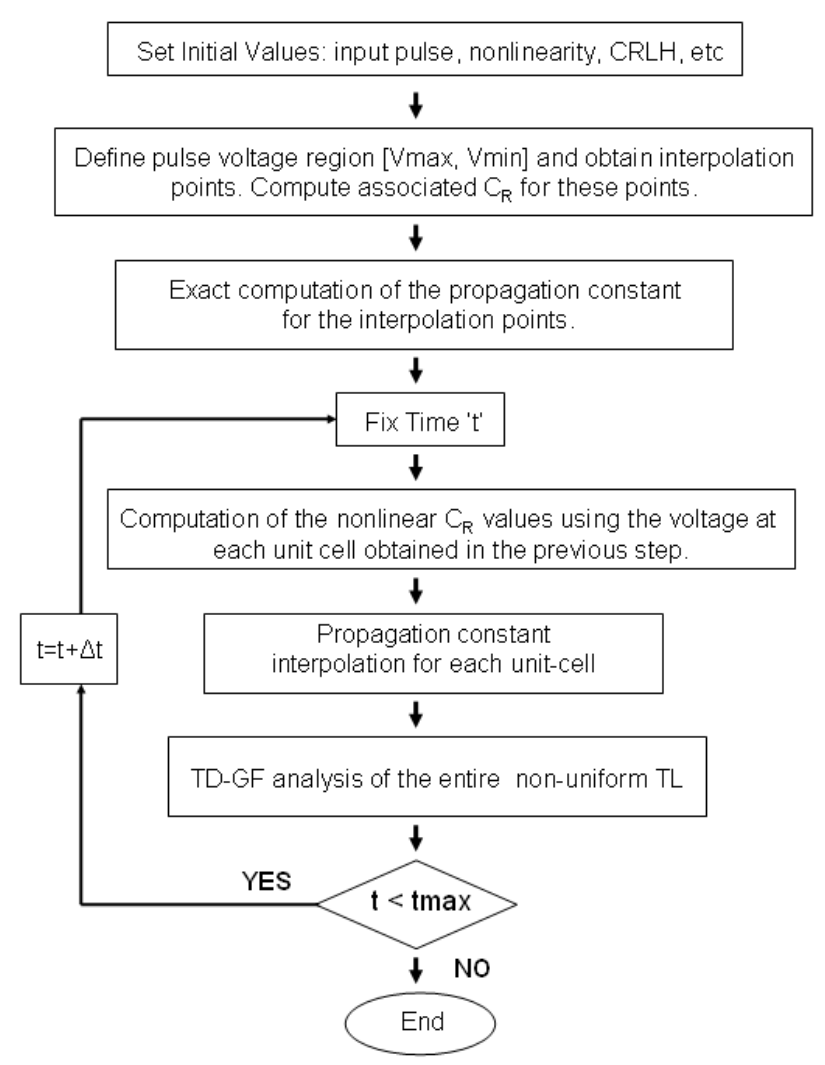

Figure 5: Flowchart of the proposed non-linear time-domain Green's function approach.

The proposed method is able to model pulse propagation taking into account two different phenomena, dispersion and non-linearity. In order to show the practical value of the proposed technique, we feed a modulated Gaussian pulse (with $f_{0}=2.5 \mathrm{GHz}$, $V_{0}=1 \mathrm{~V}$ and $\sigma_{0}=0.4 \mathrm{~ns}$ ) into a non-linear CRLH line (composed of 48 unit cells, with unit length equal to $p=1.56 \mathrm{~cm}$, circuital parameters $C_{0}=C_{L}=1.0 \mathrm{pF}$ and $L_{R}=L_{L}=2.5 \mathrm{nH}$ and non-linear parameters $\left.\eta=\alpha=8 \cdot 10^{-13}\right)$. This configuration may easily be implemented (for instance, in microstrip technology) and provides both, dispersion and non-linearity phenomena.

Fig. 6a) presents the temporal output waveform of the pulse, computed with the proposed method and validated with the commercial software ADS. As can be observed, good agreement is achieved. Fig. 6b) depicts the spectrum waveform of the pulse, at the position of $z=0.7 \mathrm{~m}$ (i.e. in the middle of the non-linear CRLH line), obtained by both, the proposed technique and ADS. Again, the agreement between the two different methods is good. In the figure, pulse generation at a frequency multiple of the input pulse is clearly visible, confirming the non-linear behaviour of the line. In Fig. 6c) the temporal propagation of the input pulse (envelope) along the structure is shown. It is interesting to observe the reflected waves along the media, produced by the characteristic impedance fluctuations with time at each unit cell. The pulse spreading, due to the dispersive features of the line, is clearly visible. Fig. 6d) presents the spectrum evolution of the pulse along the medium, showing the harmonic generation. Note that the low frequencies of the input pulse have been filtered out by the band-pass behaviour of the CRLH line. The explanation of the frequency variations along the line is as follows. Initially, the pulse enters into the non-linear CRLH with a maximum amplitude of $V_{0}$, which creates a moderate impedance discontinuity (due to the Bloch impedance variation). This creates reflected waves, as previously commented. In 
addition, this initial amplitude $V_{0}$ also generates harmonics with a maximum of amplitude at the beginning of the line. Then, as long as the pulse is propagating though the medium, the amplitude of the pulse is decreasing, due to the dispersion, consequently decreasing the amplitude of the generated harmonics. Note that an interference pattern occurs in the last CRLH-PRH transition, due to a small temporaldependent impedance mismatch.

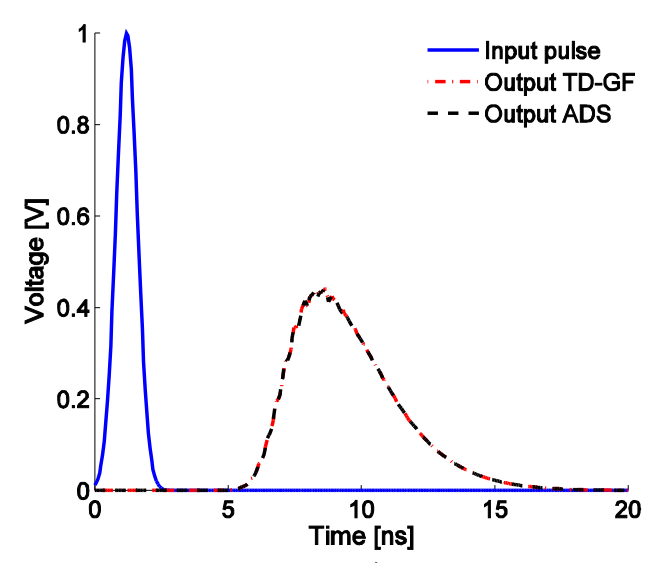

a)

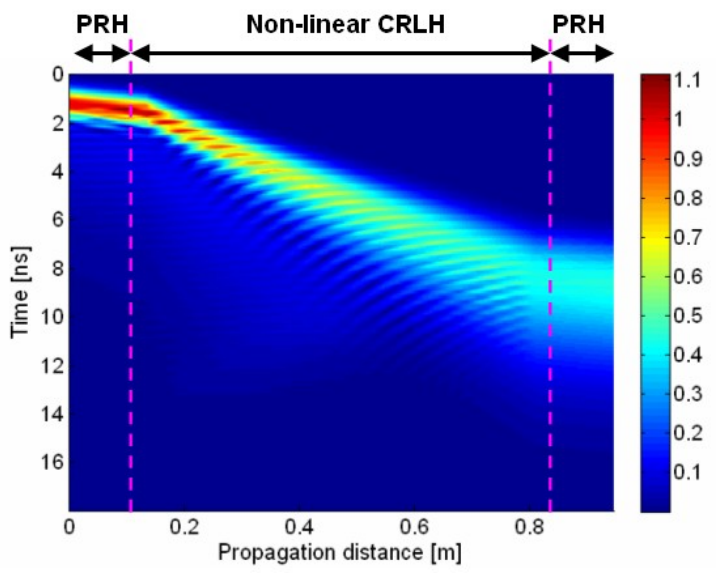

c)

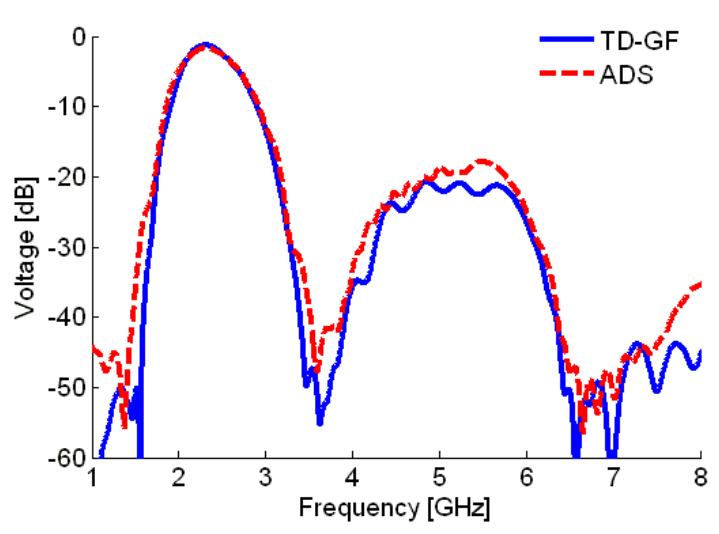

b)

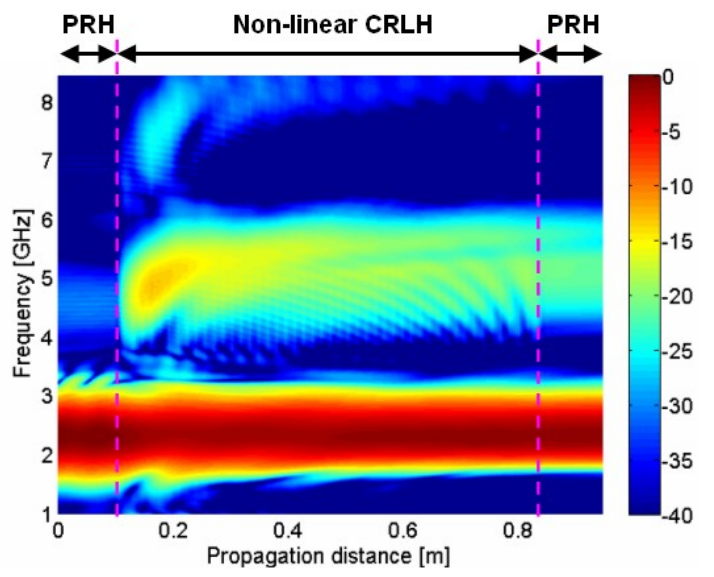

d)

Figure 6: A modulated Gaussian pulse ( $f_{0}=2.5 \mathrm{GHz}$ and $\sigma_{0}=0.4 \mathrm{~ns}$ ) is fed into a non-linear CRLH TL (48 unit cells, with unit length equal to $p=1.56 \mathrm{~cm}$, circuit parameters $C_{0}=C_{L}=1.0 \mathbf{p F}, L_{R}=L_{L}=2.5 \mathrm{nH}$ and non-linear parameters $\eta=\alpha=8 \cdot 10^{-13}$ ) sandwiched with two conventional right-handed lines. (a) Output waveform (only enveloped shown) provided by the proposed method and validated with ADS $\odot$. (b) Spectrum waveform provided by the proposed method and validated with ADS $\odot$, at the distance $z=0.7 \mathrm{~m}$. (c) Propagation of the input pulse (envelope) along the lines. (d) Spectrum evolution of the pulse along the lines.

Finally, we present in Table 1 a comparison, between the original and interpolated method, for the time required to compute the results presented in Fig. 6. For this computation, we have employed a total of 600 time steps (between 0 and $18 \mathrm{~ns}$ ) and we have analyzed a total number of 2000 spatial points, uniformly distributed along the 3 mediums (2 PRH and 1 non-linear CRLH composed of 48 unit-cells). Note that the exact propagation constant has been calculated just for 11 values of $C_{R}$, at each frequency, in order to provide accurate data for the interpolation. These capacitors values are computed following Eq. (12), employing as an input voltage discrete values from a uniform discretization of the input pulse amplitude range. The time required for 
this computation has already been included in Table 1. In Fig.7, the maximum relative error during the propagation constant computation, for different values of frequency, is presented. As can be observed in the figure, the maximum error achieved with the interpolated method is always below $0.1 \%$, due to the very smooth behaviour of the CRLH propagation constant under weak non-linear conditions. Thereby, the use of the interpolated method is justified, providing an $80 \%$ reduction of the computational cost while maintaining high accuracy.

\begin{tabular}{|c|c|c|}
\hline $\begin{array}{c}\text { Original Method } \\
\text { Time [seconds] }\end{array}$ & $\begin{array}{c}\text { Interpolated Method } \\
\text { Time [seconds] }\end{array}$ & $\begin{array}{c}\text { Improvement } \\
(\%)\end{array}$ \\
\hline 325.57 & 65.12 & $\approx 80.0 \%$ \\
\hline
\end{tabular}

Table 1: CPU-time comparison for the pulse propagation computation along a non-linear CRLH, obtained with the original and with the interpolated schemes.

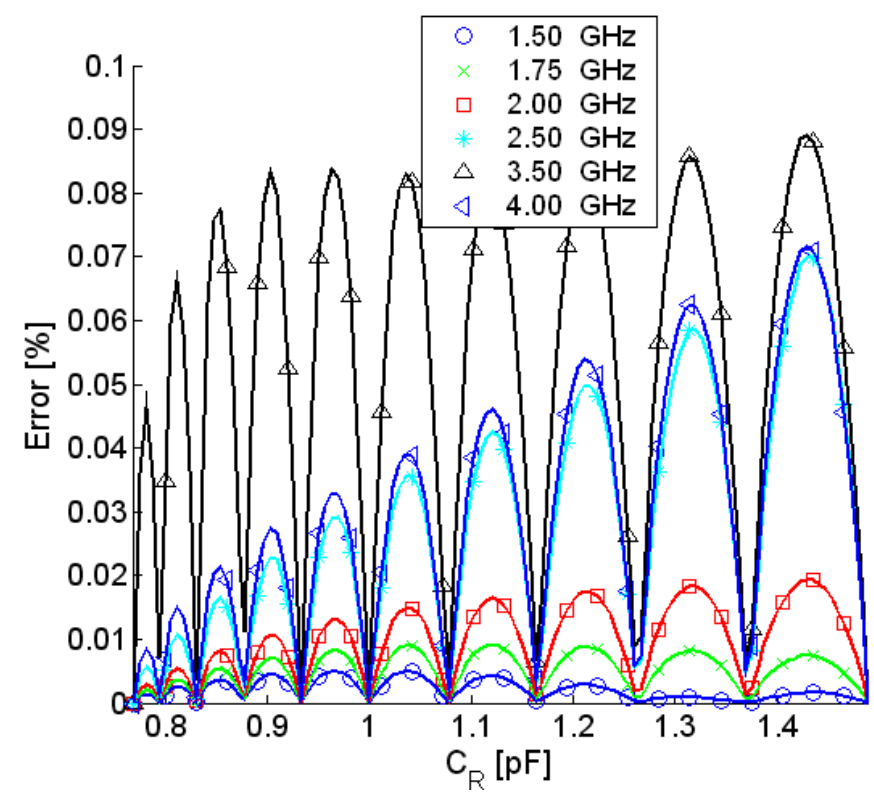

Figure 7: Relative error between the original and interpolated calculation of the propagation constant, versus $C_{R}$ for different frequencies.

\section{Radiating Metamaterial Structures}

The use of composite right/left-handed (CRLH) structures as leaky-wave antennas (LWA) was introduced with full-space scanning capabilities in [3]. Specifically, the propagation constant of the CRLH structure penetrates into the fastwave region [20], and the structure behaves as a leaky-wave antenna, radiating from backfire $\left(\boldsymbol{\theta}=-90^{\circ}\right)$ to endfire $\left(\boldsymbol{\theta}=90^{\circ}\right)$, including the broadside direction $\left(\boldsymbol{\theta}=0^{\circ}\right)$. The mapping between each frequency within the fast-wave region into a specific radiation angle follows the LWA beam-scanning law [20]:

$$
\theta_{M B}=\sin ^{-1}\left[\frac{\beta(\omega)}{k_{0}}\right]
$$


where $\theta_{M B}$ is the radiation angle and $k_{0}$ is the free space wavenumber.

Based on this spectral-spatial decomposition, a novel real-time spectrogram analyzer (RTSA) has recently been proposed $[15,16]$. The idea is to characterize, both in frequency and time, an unknown UWB input signal. The system is composed of a CRLH LWA and a number of probes located in a semi-circular configuration in the farfield region of the antenna (see Fig. 8). The description of the system is as follows. Initially, an unknown broadband signal under test is used to excite the CRLH LWA. The different spectral components of the signal are radiated, at each particular instant, to different directions in space. Then, each probe receives the temporal evolution of a particular frequency of the input test-signal. Note that the frequency received by each probe depends on the particular position of the probe in space, following Eq. (15). Finally, the time information of all probes is combined in order to obtain a spectrogram [21], which provides a joint time-frequency representation of the unknown signal.

In order to analyze this system, full-wave commercial software, such as CST, may be employed. However, the generation of these results are extremely timeconsuming due to the complexity of the system and the temporal nature of the analysis. An interesting alternative is to extend the formulation of the efficient time-domain Green's function to model the CRLH LWA radiation as a function of time.

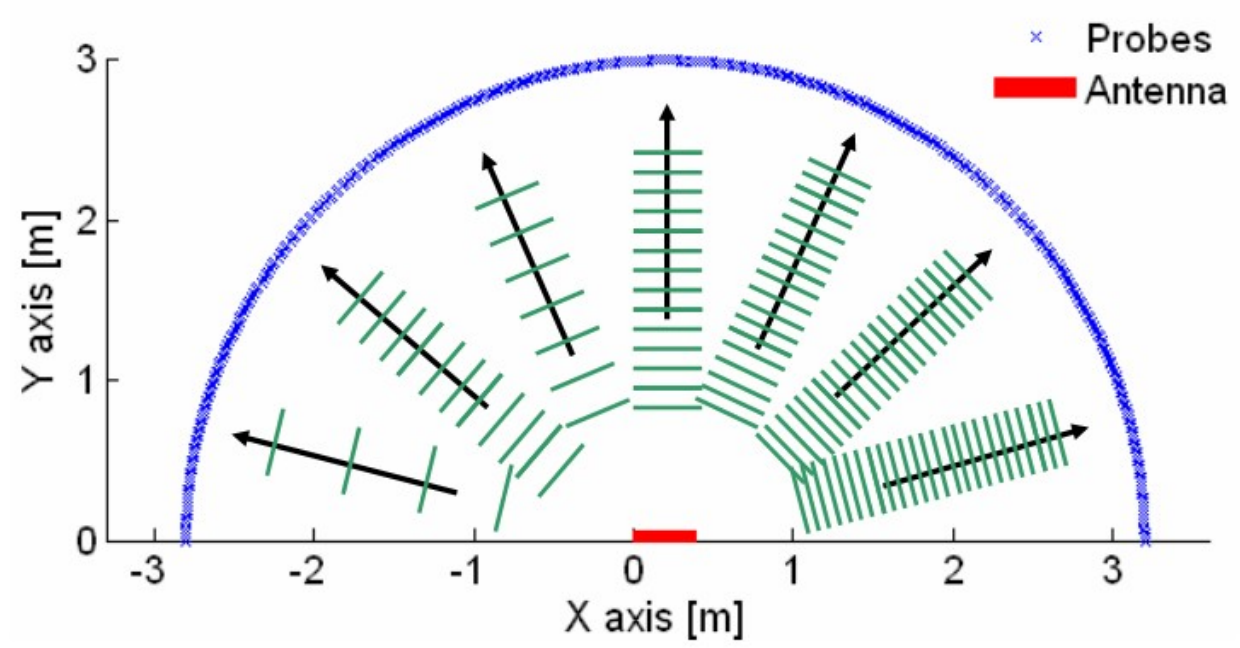

Figure 8: CRLH Leaky-wave antenna configured as a real-time spectrogram analyzer (RTSA) [15, 16], and 181 far-field probes arranged in a semi-circular configuration to receive the transmitted signal. The CRLH structure is composed of 32 unit cell, with unit length equal to $p=1.0 \mathrm{~cm}$, and with circuit parameters $C_{R}=C_{L}=1.0 \mathrm{pF}$ and $L_{R}=L_{L}=2.5 \mathrm{nH}$.

Consider, for this purpose, a single CRLH leaky-wave antenna [see Fig. 1 a)], which is fed by a point source at the position $\vec{r}_{g e n}$. The generator produces a temporal input signal, which propagates along the CRLH transmission line and induces a current along the structure. This current can be computed combining Eq. (2) with Eq. (4). Once the current distribution along the antenna is known for all frequencies in the fast-wave region, the corresponding far-field radiation as a function of time is obtained using standard Fourier transforms [22]. This far-field radiation is finally given by 


$$
E(\vec{r}, t)=-j \frac{\mu_{0}}{2 \pi} \iint_{z_{0}}^{z_{\ell}} \omega \tilde{G}_{I}\left(z^{\prime}, z_{g} ; \omega\right) \tilde{I}(\omega) \frac{e^{-j k_{0} R}}{R} \partial z^{\prime} \partial \omega
$$

where $\vec{r}$ is the observation point in free-space, $z_{0}$ and $z_{\ell}$ are the initial and last points of the physical structure (with $\ell=z_{\ell}-z_{0}$ ), $z^{\prime}$ is an arbitrary point along the CRLH line and $R=\left|\vec{r}-z^{\prime}\right|$.

This method can be efficiently employed to characterize an RTSA system. As a general example, consider a CRLH LWA composed of 32 unit cells of length $p=1.0$ $\mathrm{cm}$, with circuit parameters $C_{R}=C_{L}=1.0 \mathrm{pF}$ and $L_{R}=L_{L}=2.5 \mathrm{nH}$. To complete the system, a total number of 181 observation probes are placed in a semi-circular configuration, as shown in Fig. 8. The first step to model the RTSA is to perform the calibration of the system. This is necessary to compensate for the different power levels received at each probe $[15,16]$, due to the directivity variation with frequency [3]. For the calibration, a narrow-band signal is modulated to the different fast-wave frequencies [following Eq. 15] and subsequently radiated by the LWA. Then, the maximum power received at each probe is stored, obtaining a normalization rule for this particular system configuration. In our particular example, the calibration result is shown in Fig. 9.

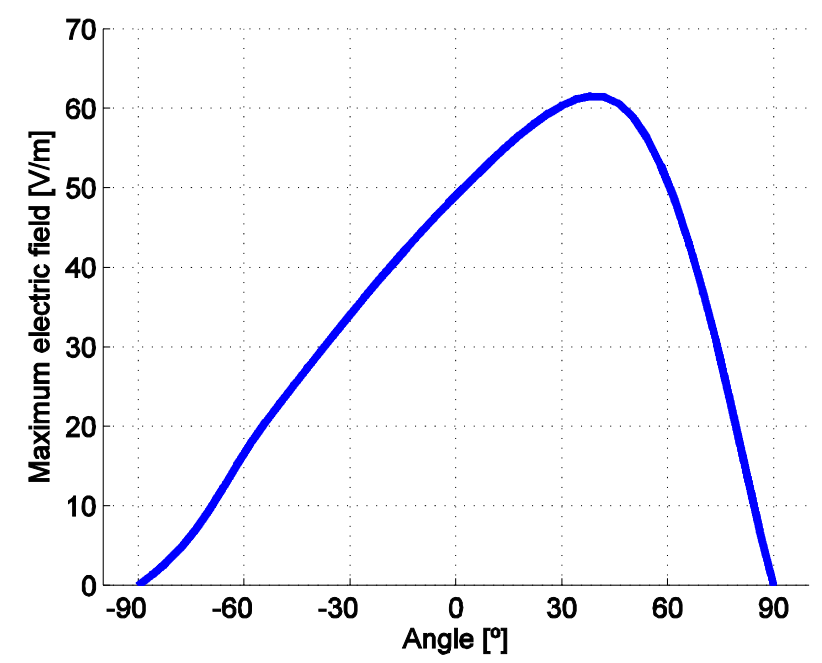

Figure 9: Maximum electric field obtained at the different positions of the probes, used for the calibration of the RTSA system of Fig. 8.

Once the system has been calibrated, it can be efficiently used to obtain spectrograms of an unknown input signal. It is important to mention the reduced time needed for the analysis, requiring about 30 seconds on a regular PC, as compared to full-wave simulations $[15,16]$, which require several hours. For the method validation, we use the real temporal and frequency information of the input signal, which is known in the numerical simulation.

In the first example, the CRLH LWA is fed by a signal composed of three modulated Gaussian pulses. The first pulse has a positive-chirp modulation (which means that the modulation frequency is increasing with time), and the third pulse has a negative-chirp modulation. The spectrogram obtained with the proposed method, after calibration, is depicted in Fig. 10, including an additional graph showing the analytical 
temporal representation of the signal. As can be observed in the figure, the spectrogram follows the signal variations in time (the three pulses are clearly observable) and also, simultaneously, in frequency. It is especially interesting to observe the transition between two consecutive pulses, where frequencies corresponding to different pulses appear at the same instant.

In the second example, a self-phase modulated pulse [8] is employed to feed the CRLH LWA. This pulse has strong variations in frequency, as show in Fig. 11. The spectrogram has been obtained using the formulation presented, initially without the power compensation [see Fig. 11 a)] and then introducing this correction [Fig. $11 \mathrm{~b}$ )]. As can be seen in the figures, the calibration system correctly modifies the spectrogram levels, accurately characterizing the input pulse both in frequency and time. Note that the antenna length plays an important role to frequency discrimination, because it is related to the antenna directivity. A larger antenna, with increased directivity, will also be able to follow the faster frequency variations of the pulse.

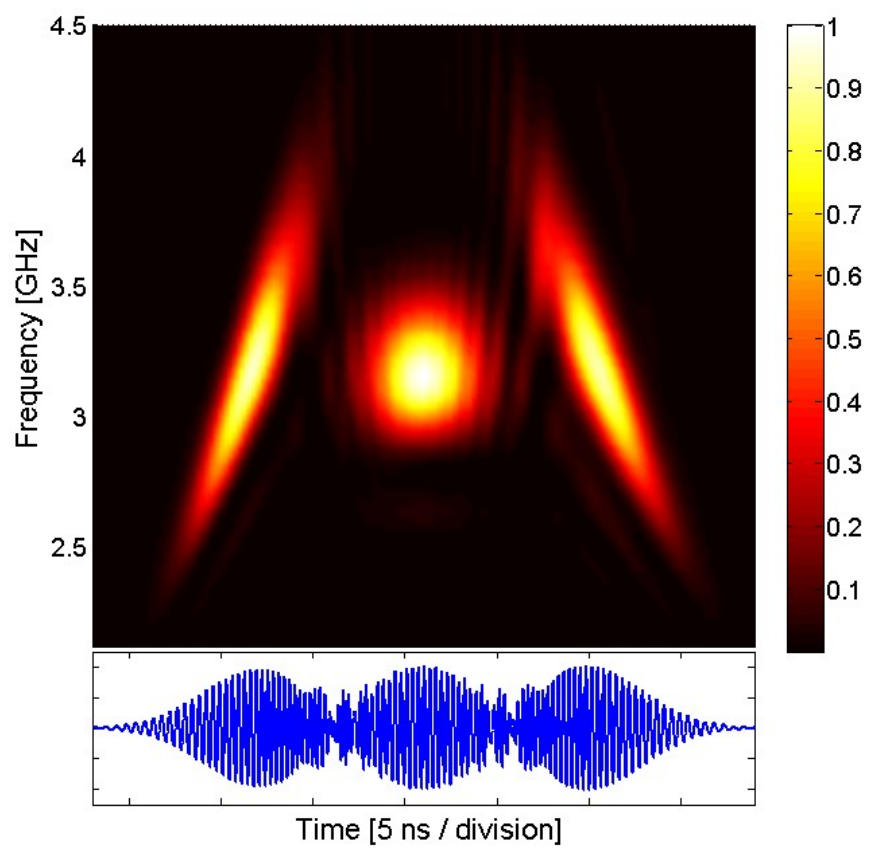

Figure 10: Normalized spectrogram of a three chirp-modulated Gaussian pulses, with chirp parameters $\mathbf{C}=-[10, \mathbf{0}, 10]$, modulation frequency $f_{0}=3.19 \mathrm{GHz}$ and temporal width $\sigma=1.0$ ns, computed with the proposed technique. The inset shows the analytical time response of the signal.

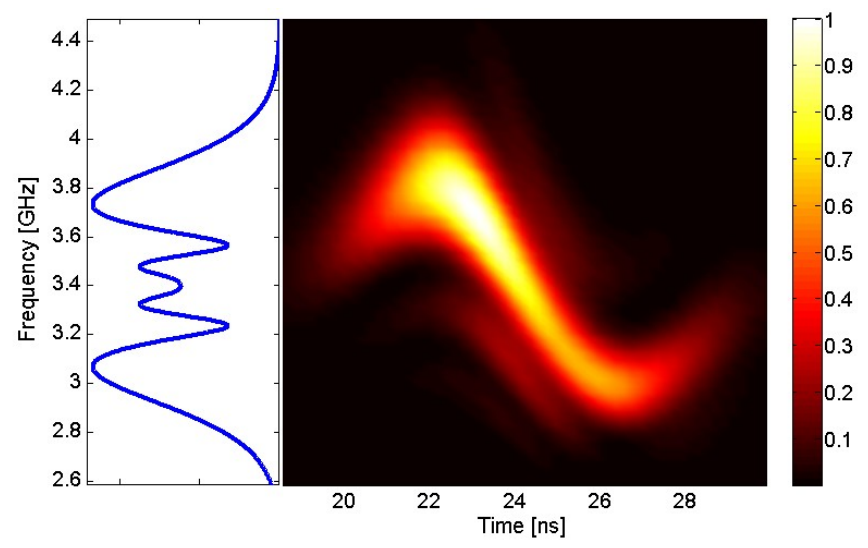


a)

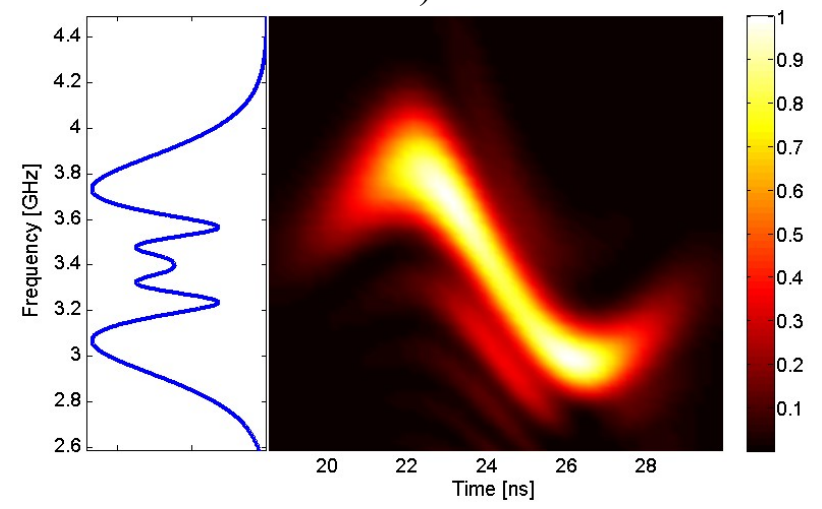

b)

Figure 11: Normalized spectrogram of a self-phase modulated pulse (SPM), with $f_{0}=3.4 \mathrm{GHz}$, $m=1, z=10$ and $\sigma=8$ ns [following the notation of 16]. The inset shows the analytical frequency response of the signal. (a) Without power calibration. (b) Including power calibration.

The main advantage of the RTSA system is its analogue nature, able to provide real-time spectrogram results in practice. The method proposed here is able to perform a quick and accurate modelling of the system, with deep insight into the CRLH LWA time-radiation properties, and avoiding the extremely time-consuming analysis required in full-wave simulations. Therefore, it provides a fast tool to configure an RTSA system and to determine a priory the range of input signals which can accurately be characterized.

\section{Conclusions}

In this paper, a numerical method has been proposed for the analysis of highlydispersive linear and non-linear metamaterial structures. Initially, pulse propagation along non-linear composite right/left-handed transmission line (CRLH TL) has been carefully studied. For this purpose, a dispersive CRLH line has been loaded with hyper abrupt diodes to achieve non-linearity. The characteristic impedance and propagation constant of each unit cell of the CRLH TL is then varied at each time-step, and the nonuniform line is analyzed. A novel interpolation scheme, based on the smooth behaviour of the propagation constant with small variations of the shunt capacitor has been proposed. This scheme has provided an $80 \%$ reduction of the computational cost maintaining high accuracy. A general example which combines dispersion and nonlinearity has finally been presented, validated and discussed. Secondly, the impulseregime radiation of a leaky-wave antenna has been analyzed and employed to describe a real-time spectrogram analyzer (RTSA). A calibration method for the system, which takes into account the directivity variation of the antenna with frequency, has been introduced. Numerical results have shown that the proposed technique provides an extremely fast and accurate tool to characterize leaky-wave based RTSA systems.

\section{Acknowledgement}

This work was partially supported by the Spanish Ministry of Education and Science under Grant FPU-AP2006-015 and with the Project TEC2007-67630-C03-02. 


\section{References}

[1] M. Ghavami, L. B. Michael, and R. Kohno, "UWB Signals and Systems in Communication Engineering”. J. Wiley \& Sons, 2007.

[2] M. 1. Skolnik, Introduction to Radar Systems, 3rd ed., McGraw-Hill, 2001.

[3] C. Caloz and T. Itoh, Electromagnetic Metamaterials: Transmission Line Theory and Microwave Applications. Wiley and IEEE Press, 2005.

[4] N. Engheta and R. W. Ziolkowski, Electromagnetic Metamaterials: Physics and Engineering Explorations. Wiley-IEEE Press, 2006.

[5] J. S. Gomez-Diaz, A. Alvarez-Melcon, S. Gupta and C. Caloz, "Impulse-Regime CRLH Resonator for Tunable Pulse Rate Multiplication”, Radio Sci., 44, RS4001, July, 2009, doi:10.1029/2008RS003991.

[6] J. S. Gomez-Diaz, A. Alvarez-Melcon, S. Gupta and C. Caloz, "Spatio-Temporal Talbot Phenomenon using Metamaterial composite Right/Left-Handed Leaky-Wave Antennas”, Journal of Applied Physics, 104, 104901 (2008), doi:10.1063/1.3013905.

[7] B. E. A. Saleh and M. C. Teich, Fundamentals of Photonics, 2nd ed. Wiley-Interscience, 2007.

[8] G. P. Agarwal, Nonlinear fiber optics, Academic Press, 2005.

[9] S.Gupta and C. Caloz, "Dark and Bright Solitons in Left-Handed Nonlinear Transmission Line Metamaterials", in Proc. IEEE MTT-S Int. Microwave Symp. Dig., Honolulu, Hawaii, pp. 979-982, June 2007.

[10] A. Taflove and S. C. Hagness, Computational Electrodynamics: The Finite-Difference Time-Domain Method. Artech House, 2005.

[11] W. J. R. Hoefer, "The Transmission-line Matrix Method-Theory and Applications," IEEE Trans. Microwave Theory Tech., vol. 33, pp. 882-893, 1985.

[12] J. S. Gómez-Díaz, S. Gupta, A. Álvarez-Melcón and C. Caloz, "Investigation on the Phenomenology of Impulse-Regime Metamaterial Transmission Lines", IEEE Trans. Antennas and Propagation, December 2009.

[13] J. S. Gómez-Díaz, A. Álvarez-Melcón and C. Caloz, "Time-Domain Green’s Function Technique for Highly-Dispersive Metamaterial Waveguide and Antenna Structures" in IEEE Asia-Pacific Microwave Conference (Hong-Kong, China, 2008).

[14] A. B. Kozyrev and D. W. van der Weide, "Nonliner Wave Propagation Phenomena in Left-Handed Transmission Line Media", IEEE Trans. Microwave Theory and Techniques, vol. 53, no. 1, January 2005.

[15] S. Gupta, S. Abielmona, and C. Caloz, "Leaky-wave based spectrum analyzer with unrestricted timefrequency resolution," in Proc. IEEE MTT-S Int. Microwave Symp. Dig., Atlanta, GA, pp. 807-810, June 2008 .

[16] S. Gupta, S. Abielmona, and C. Caloz, "Microwave Analog Real-Time Spectrum Analyzer (RTSA) based on the Spatial-Spectral Decomposition Property of Leaky-Wave Structures", IEEE Trans. Microwave Theory and Techniques, December 2009.

[17] G. Barton, “Elements of Green's Functions and Propagation”. Oxford Science Publications, 1989.

[18] D. Pozar, Microwave Engineering, 3rd ed. John Wiley and Sons, 2005. 
[19] P. Russer, Electromagnetics, Microwave Circuit and Antenna Design for Communications Engineering. Artech House, 2006.

[20] A. Oliner and D.R. Jackon, "Leaky-wave antennas" in Antenna Engineering Handbook, 4rd ed. McGraw-Hil, New York, 2007.

[21] L. Cohen, "Time-frequency distributions-a review", Proceedings of the IEEE, vol. 77, no. 7, pp. 941981, July 1989.

[22] C. A. Balanis, “Antenna Theory: Analysis and Design”, Wiley-Interscience 3rd ed. 2005. 